\title{
Study of animal welfare status and heat stress measures applied in dairy cow herds in Hungary
}

\author{
Gudaj Richárd ${ }^{1}$ - Komlósi István ${ }^{1}$ - Brydl Endre ${ }^{2}$ \\ ${ }^{1}$ Centre for Agricultural and Applied Economics Science \\ Faculty of Agricultural and Food Sciences and Environmental Management \\ University of Debrecen \\ ${ }^{2}$ Department of Animal Hygiene \\ Faculty of Veterinary Science \\ Szent István University in Budapest. \\ rgudaj@agr.unideb.hu
}

\begin{abstract}
SUMMARY
The following material focuses on dairy production and climate related issues in Hungary. All the data was gathered during PhD project: Study of animal welfare status in dairy cow herds in Hungary. Relations between animal welfare and climate changes expressed by increase in temperature are described. Extremely hot weather creates hard conditions for milking cows when animal welfare is highly compromised. From the preliminary results obtained one might formulate hypothesis that there are still areas on the farms where immediate actions should be taken to give a relief to cows in hot seasons. There was found significant number of farms with too many animals per one water trough, dirty water troughs, limited access to water troughs and hazardous surface for cows in critical places where many animals are gathered. Calves with not sufficient amount of water in hot days and other parts of the year were reported. Silage exposure to the sun and mouldy food in a silage clump was also found to be an important factor in monitoring impact of warm weather. Half of the farms letting animals to spend time on the pasture or paddock did not provide shade for animals. Low conception rate of first insemination was predicted to be influenced by heat stress, what is proved by lack of heat decreasing measures taken on the farms.
\end{abstract}

Keywords: heat stress; dairy welfare, dairy welfare assessment protocol, multivariable modelling, herd health

\section{INTRODUCTION}

Changes in climatic conditions, market organisation, societal demands on food quality, animal welfare and environmental quality have created new conditions for farmers as well as for researchers and policy makers. New social, technical and economic solutions are needed for farming and rural areas. There is a wide range of measures related to dairy welfare and changes in climate. One of them is high temperature which creates heat stress. This is one of the leading causes of decreased production and fertility in dairy cattle during summer months in many parts of the globe (De Rensis and Scaramuzzi, 2003). These losses are apparent in the decreased amount of milk shipped, increased days open and decreased breedings per conception. Some heat stress is unavoidable, but effects can be minimized if certain management practices are followed. Cattle sweat at only 10 percent of the human rate, so that affects that they are more susceptible to heat stress (West, 2003). That is why dairy cattle need mechanical means to reduce heat, such as body sprinkling to aid in evaporation and effective air movement systems to aid in cooling. Stale, stagnant air can reach dangerous or lethal areas in a short time. Therefore, it is essential to have rapid movement of air in any confined area. During times of heat stress feed intake is reduce by 8 to 12 percent or more. This reduction in feed intake reduces volatile fatty acid production in the rumen, resulting in decreased production.

Welfare Quality® Assessment Protocol for Cattle (2009) is a world recognised document which covers all animal welfare measures needed to be taken in assessing welfare of dairy cattle. However, as yet, there is no measure developed for assessing thermal comfort. That emphasises how hard it is to evaluate if animals feel fine in environment with particular temperature.

The PhD project presented covers animal welfare dairy issues on Hungarian dairy farms. Climate related aspects of milk production can be clearly found as well. The main aim of the study is to use all available measures for assessing welfare of dairy cattle in Hungary. The survey tries to give the answers what are the main areas for improvement on the farms regarding animal welfare. The work was created to emphasise the importance of animal welfare in Hungary and to use the information for an academic and commercial purposes. Another aim is to communicate with farmers and people involved in dairy industry to check their knowledge and attitude to welfare issues. The final report can be presented to the public and possibilities for improvement can be discussed. 


\section{MATERIALS AND METHODS}

18 dairy farms in Hungary were recruited into the study which is in progress (target - minimum 40). The study was undertaken between June and September 2010 when extremely high temperatures were reported. The selection was firstly created on a principle of searching for as different farms as possible. On each farm measures were taken which covered 203 specific questions and critical points on the farms related to animal welfare. Animal health, behaviour, hygiene, feedstuff condition, general production data, facilities and stockman attitude were measured. Data collection was created according to already existed assessments (Whay, et al., 2003). The project, however, needed the protocol to be established again, as in Hungarian conditions some of the points of the protocol would simply not work (for example - access to the pasture all year round - which is not so popular). For the purpose of this paper climate and welfare related topics will be described only.

\section{RESULTS AND DISCUSSION}

As far the research is in progress the only results can be based on comparisons on how many farms particular conditions were found. Among measures taken there are few found to be in close relation to heat stress:

\section{Animals/water troughs}

There are many ways to decrease the apparent effects of heat on dairy cattle. One important point to consider is increasing the amount of available water which is the most significant nutrient for dairy cows. A sufficient supply of clean water is generally accepted as essential to prevent negative effects on animal health, performance, and welfare (LeJeune et al., 2001). According to Welfare Quality® Assessment Protocol for Cattle (2009) recommendations are for one water trough per 20 heads. The average number of animals per water trough in the study was 39,6 .

\section{Water quality}

Next to amount of water there is a quality of water animals are drinking. What is more, milking cows require good quality water for maintaining high milking production. This is crucial in mastitis development and digestion procedure. Algae, bacteria, protozoa, viruses and other organisms in dirty water disrupt homeostasis in organism of milking cow. One of the ways to increase water consumption is to make certain water cool. In experiment of Meyer et al. (2004) cattle increased their water consumption when the water was at $10^{\circ} \mathrm{C}$ degrees rather than $28^{\circ} \mathrm{C}$ degrees. So if the goal is to maximize intake, it makes sense to keep feed and water available in cooled areas. On some farms in the project managers provide warm water in hot days to cows. In their opinions cow are less likely to sweat if they are drinking slightly warmer water. 55\% water drinkers on the farms were found dirty.

\section{Access to water troughs}

Competition for limited resources is found when cows need to access a water trough in hot days. On many farms access to water troughs is highly limited by wrong designed facilities in barns. Problems with accessing water might cause frustration and aggressive behaviour among cows what is not recommended from production and welfare point of view. 33,3\% of farms were found with limited access to water.

\section{Surface next to the water troughs}

Many animals next to water troughs make the flooring extremely dirty. That affects lameness what is caused by slurry, muck and Treponema family bacteria developing in these conditions. Slippery floors in addition with many individuals per square meter might create a situation when uncontrolled stepping and hazardous injuries of the hooves appear. $66,6 \%$ of farms were found with dirty surface next to the water troughs.

\section{Ventilation}

Increased humidity, temperature and ammonia accumulate in areas with inadequate ventilation and are recognised as stresses for dairy cows. Ammonia dissolves in the suspended water vapour and is an irritant to the respiratory epithelium. Microscopic droplets of moisture contain bacteria and viruses which are exhaled by animals. High humidity also increases exposure to airborne organisms and increases the moisture content of bedding materials, thereby increasing the rate of bacterial growth in bedding. Adequate ventilation is important in all dairy cattle housing systems, both for cow comfort and to minimize exposure to mastitis causing bacteria. The percentage of cows standing increases as environmental temperatures increase. Standing maximizes 
evaporative cooling from body surfaces. A good air exchange will remove moisture from the building and help keep the stall and floor surfaces drier. For that reason ventilation is very important to make a relief for cows in hot seasons. What more, lack of ventilation in free stall yards gives an impression for cows the muck under the straw is warmer than it is. This is all because the fermentation and heat which is released from muck during fermentation. In these situations cows are more likely to stay than lay. The cows which are forced to stay for a long period of time are in danger of being lame quicker, because they are not providing relief for their legs. $94,4 \%$ of farms in the study are equipped with these devices.

\section{Calves with no water}

Water is the most important component of living organisms. Water is also the most important nutrient for dairy cattle. Except milk replacer calves need to have water. Fresh, clean water is essential for calf growth. Water helps with development of the rumen and the digestion of calf starter and allows earlier weaning. In the 1984, research by A.F. Kertz at Ralston Purina found that weight gain was reduced by $38 \%$ and starter intake by $31 \%$ for calves between birth and 4 weeks of age that did not receive supplemental water in addition to their milk replacer (Kirk, et al., 2002). In 44,4\% of cases in the research calves were not provided with sufficient amount of water in hot days. In these cases water and/or milk was offered two times a day.

\section{Silage exposure to the sun and mould on clamp face}

Silage quality has an influence on cows' feed intake. When cows are eating more tasty good quality food there is greater chance they will produce more milk. Exposure of silage clamp to the sun will lower quality of feed stuff. Affected silage is dry, warm and looses feeding mineral content. These conditions are preferable for development of mycotoxins and fungi. $100 \%$ of silage clamps in the study were exposure to the sun light. In average $17,5 \%$ of the whole area of the clamp face wall was observed moulded on all farms.

\section{Shade on a pasture}

It is well-known that dairy cows might suffer from heat stress when exposed to sun and high temperatures. Next to water shade is another basic resource which should be provided for living organism in hot days. $46,1 \%$ of farms letting cows to be outside of the barn did not provide shade for their cows.

\section{Conception rate to 1 st service}

The calculation is defined as percentage of successful inseminations after first service. Less inseminations needed for producing one calf means the production is more efficient. The research took place in summer months between June and September. In the study $28 \%$ of cows after first lactation were inseminated successfully. That means $72 \%$ of cows had to be inseminated more then once. There are many factors influencing reproduction of dairy cows, however heat stress could be found one of the major reasons. That thought is due to the lack of major heat measures which were observed on the farms.

\section{CONCLUSIONS}

There is a wide range of measures, problems and solutions related to climate change, mainly temperature increase. Considering all disadvantages milking industry is facing nowadays it is very easy to omit the basic needs of farm animals. From the study comes out a clear conclusion that majority of farms do not take appropriate actions do decrease negative impact of heat stress. That starts with calves with not appropriate amount of water and continues with compromised job quality performance. Farmers and farm managers are very rarely modifying the diet to maintain feed intake in hot weathers. Amount of water and shade are in many cases in the same amount like in any other parts of the year. Adaptation to climate change should start from feasible and understandable assessment of farm welfare status, particularly needs of animals and their behaviours.

\section{REFERENCES}

Blokhuis, H.J. (2009): Welfare Quality® Assessment Protocol for Cattle, 101.

De Rensis, F. and Scaramuzzi, R.J. (2003): Heat stress and seasonal effects on reproduction in the dairy cow-a review, Theriogenology, 60, $1139-1151$.

Kirk, J., Atwill, E., Holmberg, C., Arana, M., Collar, C., Ghirardelli, D., Higginbotham, G., Markagaard, G., Mullinax, D. and Wubishet, A.(2002): Prevalence of and risk factors for Salmonella in water offered to weaned dairy calves in California, USA, Preventive Veterinary Medicine, 54, 169-178. 
LeJeune, T., Besser, T.E., Merrill, N.L., Rice, D.H. and Hancock, D.D. (2001): Livestock drinking water microbiology and the factors influencing the quality of drinking water offered to cattle, Journal of Dairy Science, 84, 1856- 1862.

Meyer, U., Everinghoff, M., Gädeken, D. and Flachowsky, G. (2004): Investigations on the water intake of lactating dairy cows, Livestock Production Science, 90, 117-121.

West, J.W. (2003): Effects of Heat-Stress on Production in Dairy Cattle, Journal of Dairy Science, 86, 2131-2144.

Whay, H.R., Main, D.C.J., Green, L.E. and Webster, A.J.F. (2003): Assessment of the welfare of dairy cattle using animal-based measurements: direct observations and investigation of farm records, Veterinary Record 153, 197-202. 